\title{
Effectiveness of platelet-rich plasma (PRP) on pain, function and quality of life in knee osteoarthritis patients: a before-and-after study and review of the literature
}

\begin{abstract}
Objective: To determine the effects on pain relief, function and quality of life in knee Osteoarthritis (OA) patients by a protocol of 3-doses of PRP injected weekly.

Material and methods: A prospective quasi-experimental before-and-after study (non-randomized control trial) was performed on 27 patients with knee OA KellgrenLawrence (K-L) grade 2 or more, from January 2014 to February 2017, referred to the Rehabilitation Department at Santa Cristina's University Hospital. The symptoms severity was evaluated using the Western Ontario and Mac Master Universities Index for Osteoarthritis (WOMAC). The PRP-protocol consisted of 3-sessions (1 session/ week) of an intra articular infiltration of $3 \mathrm{ml}$ of platelet-rich plasma (PRP). To get the PRP, Accelerate Concentration System Device ${ }^{8}$ from EXACTECH, EmCyte Anticoagulant Sodium Citrate Solution U.S.P. and Drucker Centrifuge (Series Performance) were used.
\end{abstract}

Results: The mean age of the sample was $58.03 \pm 10.91$ years. Before intervention, pain measured by VAS was $8.14 \pm 1.09$ and decreased significantly to $2.96(\mathrm{p}=0.000)$ after treatment. Prior to treatment, WOMAC Pain Sub-scale was $14.88 \pm 7.02$ and decreased to $5.55 \pm 6.87$ points ( $\mathrm{p}=0.000$ ); WOMAC Stiffness sub-scale was $2.44 \pm 3.33$ and diminished to $0.4 \pm 0.4$ points $(\mathrm{p}=0.000)$; and WOMAC Function sub-scale was $44.48 \pm 12.27$ and ameliorated to $15.25 \pm 12.38$ points $(\mathrm{p}=0.000)$. As adverse effects, one patient presented important pain and inflammation after PRP infiltration (3.44\%).

Conclusion: PRP Infiltrated in a 3-doses protocol is effective in the treatment of knee OA symptoms such as pain and stiffness, improving function and QoL. PRP alleviates knee OA symptoms in all radiological knee OA grades, even the most severe ones and with statistical difference.

Keywords: Osteoarthritis, platelet-rich plasma, pain, quality of life
Volume 10 Issue 3 - 2018

\author{
Marcos Edgar Fernández-Cuadros,, \\ Susana Pérez-Moro,' María Jesús Albaladejo- \\ Florín,' Beatriz Entrambasaguas-Estepa, ${ }^{3}$ \\ Rubén Algarra-López,' \\ 'Rehabilitation Department, Santa Cristina's University Hospital, \\ Spain \\ ${ }^{2}$ Rehabilitation Department, Santísima Trinidad General \\ Foundation's Hospital, Spain \\ ${ }^{3}$ Rehabilitation Department, La Princesa University Hospital, \\ Spain
}

Correspondence: Dr. Marcos Edgar Fernández-Cuadros, Calle del Ansar, 44, piso Segundo, CP: 28047-Madrid, Tel 620-3I4558, Email marcosefc@hotmail.com

Received: May 01, 2018 | Published: May 25, 2018

\section{Introduction}

Osteoarthritis (OA) is the most common joint disease ${ }^{1}$ and produces such incapacity on the autonomy of older people, that their quality of life (QoL) is severely affected. ${ }^{2} \mathrm{OA}$ affects the QoL in its physical, emotional and social aspects, causing a great impact on the suffering and in the use of health resources. ${ }^{3}$ Almost 4 million people in Spain suffer OA and it is the cause of $50 \%$ total disability. The economic impact is such that the direct OA cost in Spain is 4,738 million Euros per year. ${ }^{1}$

In knee OA, the severity of symptoms such as pain and the loss of function lead us to look for effective treatment. ${ }^{4-6}$ To date, there is no effective cure for OA. Main goals are to reduce pain, rigidity and swelling, and to improve function on the short term. On the long-term, the objectives are to reduce joint damage and to decrease destruction of the joint. ${ }^{1,7}$

Although knee replacement provides an effective solution for severe knee OA, ${ }^{8}$ and a $95 \%$ survival rate at 10 years, ${ }^{3}$ in the case of younger and middle-aged patients and with earlier OA stages, conservative non-surgical interventions have been proposed to treat the painful joint. ${ }^{9-11}$ Conservative treatment includes analgesics, steroid and nonsteroidal anti-inflammatory drugs (NSAIDs), steroid and Hyaluronic Acid injections, ${ }^{6}$ and even Ozone injections. ${ }^{7,12}$ These agents are beneficial on the short-term but they are not effective to modify knee OA progression. ${ }^{9,10,13}$ With the intention to slow OA progression, many modalities have been used, from oral chondroprotection and intraarticular steroids, to viscosupplementation, but the outcomes are very variable. ${ }^{14}$ Moreover, there is no drug capable of inducing clinical and relevant chondroprotective properties..$^{15,16}$ Nevertheless, FernandezCuadros et al have stated that intra articular Ozone injection could slow knee OA progression on a 2 years follow-up period. ${ }^{12}$ Since there is inconclusive evidence to recommend for or against corticosteroid and strong evidence against Hyaluronic Acid viscosupplementation injections in the treatment of symptomatic knee OA, ${ }^{17}$ it is necessary to look for the emergence of some other injectable options useful to improve function and pain relief in knee OA patients. ${ }^{18}$ Knee OA is a chronic and multifactorial condition ${ }^{1,19}$ where inflammation plays an important role. ${ }^{2}$ Knee OA starts with the destruction of articular cartilage which leads to joint space lost and formation of peripheral 
osteophytes. ${ }^{1,2,12,19}$ Since there is no definitive cure to stop articular cartilage degeneration, ${ }^{19}$ recent studies have suggested possible benefits from intra-articular cell therapy. ${ }^{20}$

In the last decade, the use of new active molecules or biological compounds for the management of orthopedic disorders involving tendons, ligaments, bone and cartilage has increased exponentially and significantly. ${ }^{21}$ In particular, platelet-rich plasma (PRP) and stem cells have gained attention and popularity because of its potential properties for healing and modulation of pain and inflammation. ${ }^{21}$ PRP has become an increasingly used treatment in the field of orthopedics and sports medicine, although its primary applications began in cardiovascular and maxillofacial specialties. ${ }^{14,16,22}$

PRP is autologous plasma that after activation by exogenous agents, releases mediators (cytokines) and growth factors that increase the healing of different tissues such as ligaments, tendons, muscles and bones. ${ }^{6,16,18,23}$ Growth factors promote angiogenesis, recruitment of stem cells and fibroblasts, which induce modulation of inflammation and inhibition of catabolic enzymes and cytokines. ${ }^{6}$ Thus, PRP directly injected at the site of cartilage lesion induces a natural healing and repair of affected tissues. ${ }^{6}$ These combined effects of PRP make it a potential injectable option for the management of knee OA. ${ }^{18}$

Today, there are several trials trying to determine if PRP ameliorates OA symptoms if administered at the site of cartilage lesion by local injection or during orthopedic procedures, but the results are controversial. ${ }^{16,22,24,25}$ However, multiple studies have demonstrated efficacy in a wide variety of challenging conditions, from tendinopathy to OA. ${ }^{22}$ The controversial results of PRP in musculoskeletal pathology including tendon, bone and cartilage repair are due to heterogeneous PRP preparation methods, application techniques, outcome measures and low-level studies; then no clear conclusions can be obtained. ${ }^{23}$

The objective of this quasi-experimental observational study is to determine if whether a homogeneous protocol of 3-doses of PRP, injected weekly, is capable to improve pain relief, function and quality of life in knee OA patients in general and by different levels of knee OA severity.

\section{Material and methods}

A prospective quasi-experimental before-and-after study is been performed on 27 out of 28 patients with knee OA Kellgren-Lawrence (K-L) grade 2 or more (Figure 1). The study period run from January 2014 to February 2017 and involved Rehabilitation patients from Santa Cristina's University Hospital. Patients were referred from Traumatology, Rheumatology and Familial Medicine specialists and received non-surgical conservative treatment for at least 6 months without success (analgesics, NSAIDs, steroidal/hyaluronic acid/ Ozone infiltrations, viscosupplementation and/or physical medicine). The research was accepted by the ethical Committee of Santa Cristina's University Hospital.

\section{Inclusion criteria}

1) patients with knee OA K-L Score 2 or more; 2 ) with pain greater than 3 on VAS Scale; 3 ) older than 18 years of age; 4) who have failed any other conservative treatment (analgesics, NSAIDs, steroidal/ hyaluronic acid/Ozone injections, viscosupplementation and/or physical medicine); 5) unwilling or not available for knee arthroplasty replacement..$^{1-3,7,12}$

\section{Exclusion criteria}

1) thrombocytopenia or any severe coagulopathy: 2) any other severe disease and/or cardiovascular instability; 3) unfilled questionnaires (VAS or WOMAC); 4) absence of an informed consent; 5) patients who failed to complete the whole 3-doses PRP protocol. $^{1-3,7,12}$

At initial evaluation, demographic data was registered. An explanation of the PRP-protocol with indications and contraindications was made. Informed consent was provided and signed, and Visual Analogical Scale (VAS) and WOMAC (Western Ontario and Mc Master Universities Index for Osteoarthritis) validated scales were filled prior to treatment (Figure 1).

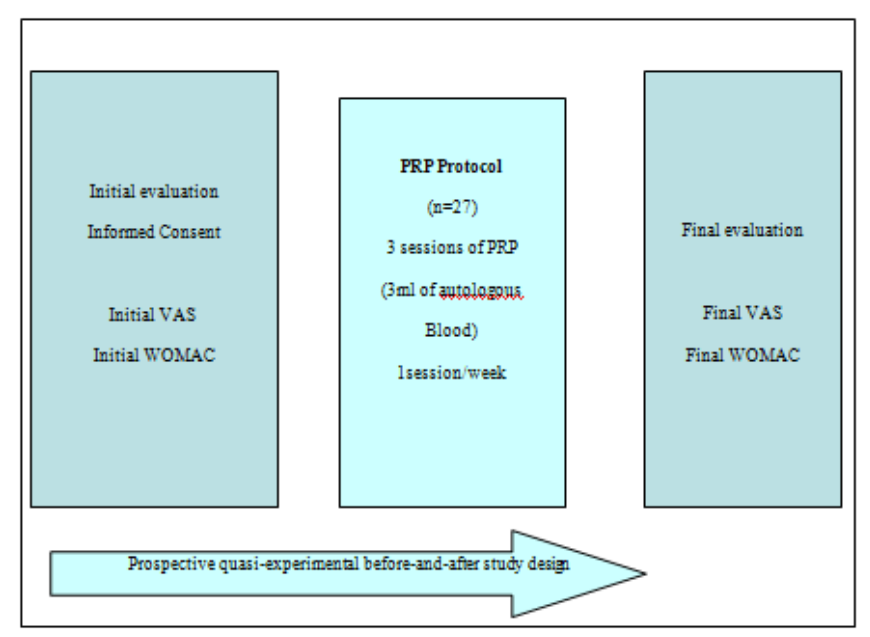

Figure I Study design.

VAS, Visual analogical scale; WOMAC, Western ontario and Mc master university index for osteoarthritis; PRP, Platelet-rich plasma

Protocol applied at the Department of Rehabilitation at Santa Cristina's University Hospital. The study ran from January 2014 to February 2017.

The proposed intervention consisted of 3 -sessions ( 1 session/ week) of an intra articular infiltration of $3 \mathrm{ml}$ of autologous blood, composed mainly on platelet-rich plasma. To get the PRP, Accelerate Concentration System Device ${ }^{\circledR}$ from EXACTECH, was used. Main steps were based on blood draw, blood processing and blood infiltration.

The Accelerate II Platelet concentrating System PRP-S $30 \mathrm{ml} \AA$ is a concentrating System that permits the rapid preparation of plateletrich plasma from $30 \mathrm{ml}$ of the patient's whole blood drawn at the time of treatment. The details of the technical procedure have been described previously by our study group (Figure 2) (Figure 3).

After PRP processing, $3 \mathrm{ml}$ were infiltrated into the lateral aspect of the flexed knee. Patients were infiltrated unilaterally on the most symptomatic knee. Previous to infiltration, $1 \%$ antiseptic chlorhexidine solution was used. After 3 sessions were performed, a clinical evaluation by VAS and WOMAC scales was performed two months after treatment, and adverse effects (if any), were recorded.

The symptoms severity of knee OA was evaluated using the WOMAC scale. ${ }^{26-28}$ The WOMAC scale is an Index that evaluates pain, stiffness and function, all in 24 items. ${ }^{26-28}$ Possible options are none, mild, moderate, severe and extreme. Pain includes 5 items 
(graded from 0-20), stiffness 2 items (scored from 0-8) and function 17 items (graded from $0-68$ ). ${ }^{26-28}$
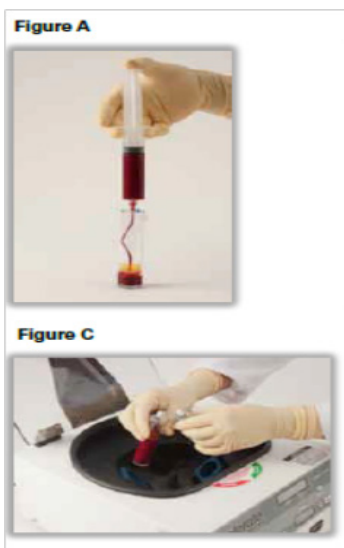

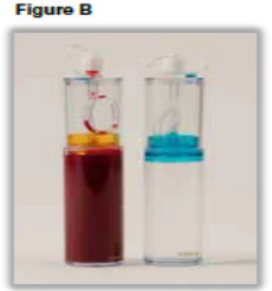

Figure D

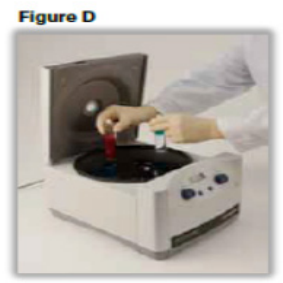

Figure 2 PRP processing. (A) Draw blood and fill into the device, (B) balance, (C) counterbalance, (D) spin the blood
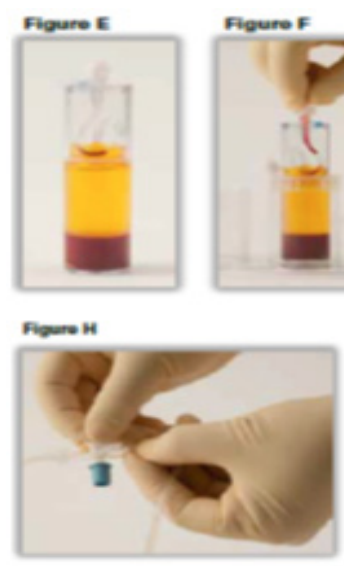
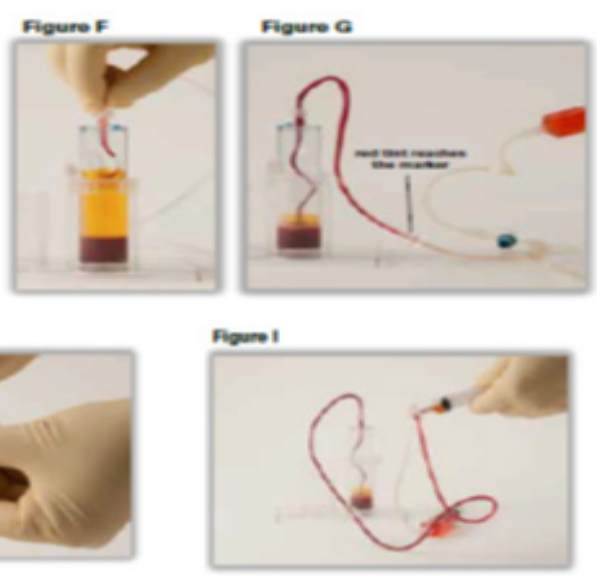

Figure 3 PRP aspiration. (E) Place the device in the holder, $(F)$ attach the transfer line to the device, $(\mathrm{G})$ to aspirate the plasma into the syringe, and stop when the red tint (buffy coat) reaches the marker. $(H)$ Once the red tint (buffy coat) reaches the marker, turn the stopcock valve "off" towards the $30 \mathrm{ml}$ plasma aspirating syringe, (I) remove the cap and attach a $12 \mathrm{ml}$ syringe to the side line connector, and aspirate $3 \mathrm{ml}$ of PRP

The knee OA radiographic severity was assessed by KellgrenLawrence (K-L) grading system, which evaluates radiographic signs. ${ }^{26,29,30}$ The K-L scale is graded as: Grade 0, no features of OA; Grade 1, small osteophytes of doubtful importance; Grade 2, definite osteophyte; Grade 3, definite osteophytes plus decreased joint space; Grade 4, definite osteophytes with important joint space reduction, sclerosis and subchondral cysts..$^{29,30}$

If WOMAC scores decrease more than $6 \%$, this difference is considered as clinically important. That means a decrease in WOMAC pain of 1.2 points; in WOMAC function of 4,1 points; and in WOMAC stiffness of 0,5 points. ${ }^{31}$

We used SPSS ${ }^{\circledR}$ version 20.0 for statistical evaluation. Quantitative variables were analyzed by means and standard deviations. Qualitative variables were evaluated by frequencies and percentages. To determine if a change for quantitative variables was significant, the t-student test was used. For the evaluation of qualitative variables (if needed), $x^{2}$ test was used. The level of significance was $95 \%$ with a $\alpha$-error of $0.5(\mathrm{p}<0.05)$.

\section{Results}

In this study, 27 out of 28 patients were studied. Fourteen patients were female $(51.8 \%)$, while 13 patients were male $(48.2 \%)$. The mean age of the sample was $58.03 \pm 10.91$ years (Table 1). The most Kellgren-Lawrence (K-L) OA grade was $3^{\circ}$ grade $(\mathrm{n}=16 ; 59.2 \%)$, followed by $4^{\circ}$ grade $(n=7 ; 25.9 \%)$ and $2^{\circ}$ grade $(n=4 ; 14.9 \%)$ (Table 1). If a relation is observed between age and OA severity, older patients present worse knee OA K-L grades. That is, for $2^{\circ}, 3^{\circ}$ and $4^{\circ}$ grades, ages were 56.6, 57.31 and 60.57 years respectively (Table 1). Table I Principal demographical and clinical variables analyzed in the sample $(n=27)$.

\begin{tabular}{ll}
\hline Variable & Value analyzed \\
\hline Female (n; percentage) & $14 ; 51.8$ \\
Male (n; percentage) & $13 ; 48.2$ \\
Ratio male:male & $1: 1$ \\
Age (years \pm SD) & $58.03 \pm 10.91$ \\
OA K-L 2 ${ }^{\circ}$ Grade (years \pm SD) & $56.5 \pm 8.54$ \\
OA K-L $3^{\circ}$ Grade (years \pm SD) & $57.31 \pm 11$ \\
OA K-L 4 $4^{\circ}$ Grade (years \pm SD) & $60.57 \pm 12.89$ \\
OA K-L 2 ${ }^{\circ}$ Grade (n; percentage) & $4 ; 14.9$ \\
OA K-L 3 ${ }^{\circ}$ Grade (n; percentage) & $16 ; 59.2$ \\
OA K-L 4 ${ }^{\circ}$ Grade (n; percentage) & $7 ; 25.9$ \\
VAS score $\pm S D(0-10)$ & $8.14 \pm 1.09$ \\
WOMAC pain score $\pm S D(0-20)$ & $14.88 \pm 7.02$ \\
WOMAC stiffness score $\pm S D(0-8)$ & $2.44 \pm 3.33$ \\
WOMAC function score $\pm S D(0-68)$ & $44.48 \pm 12.27$ \\
\hline
\end{tabular}

OA, Osteoarthritis; K-L, Kellgren-Lawrence; SD, Standard deviation;VAS,Visual analogical scale;WOMAC, Western ontario and $\mathrm{mc}$ master universities index for osteoarthritis

Before PRP-protocol treatment, pain measured by VAS was $8.14 \pm 1.09$ and decreased significantly to $2.96(\mathrm{p}=0.000)$ after treatment (Table 2) (Figure 4). Prior to treatment the WOMAC Pain Subscale was $14.88 \pm 7.02$ and decreased to $5.55 \pm 6.87$ points $(\mathrm{p}=0.000)$; WOMAC Stiffness sub-scale was $2.44 \pm 3.33$ and diminished to $0.4 \pm 0.4$ points $(\mathrm{p}=0.000)$; and WOMAC Function sub-scale was $44.48 \pm 12.27$ and ameliorated to $15.25 \pm 12.38$ points $(\mathrm{p}=0.000)$ (Table 3) (Figure 4) (Figure 5). An important amelioration of pain measured by VAS scale and a recovery in stiffness and function in all WOMAC sub-scales were observed

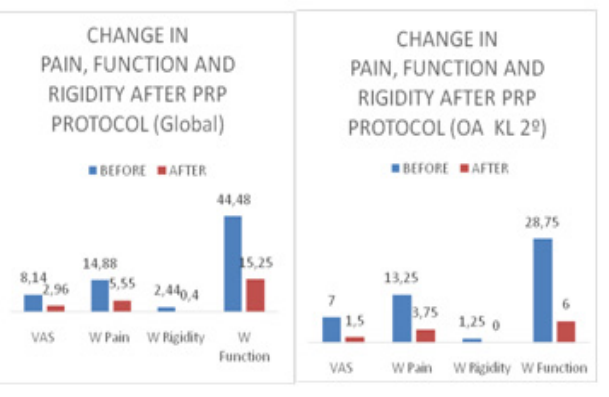

Figure 4 Change in pain, function and rigidity after PRP protocol globally and on $2^{\circ}$ grade knee Osteoarthritis.

PRP, Platelet-rich plasma; OA, Osteoarthritis; K-L, Kellgren-Lawrence; VAS, Visual analogical scale; W, WOMAC, Western ontario and Mc master universities index for osteoarthritis; WP,WOMAC pain.WR,WOMAC rigidity; WF,WOMAC function 
When pain, stiffness and function (evaluated by VAS and WOMAC) are related to knee OA severity (measured by K-L grading system), a linear relation is observed between those variables. That is, greater the severity, greater the pain and stiffness and lower the function (Table 2) (Table 3). PRP alleviates symptoms in all of OA grades and with statistical difference $(\mathrm{p}<0.05)$ (Table 2) (Table 3). PRP decreases rigidity in patients with $\mathrm{OA} \mathrm{K}-\mathrm{L} 2^{\circ}$ grade from $1.25 \pm 1.25$ points to 0 points, although this difference is clinical (minimal clinical important difference or MCID $>0.5$ points) but not statistical $(p=0.1411)$ (Table 2). As adverse effects, only one patient presented important pain and inflammation after first PRP infiltration, and he decided not to continue PRP protocol (3.44\%).

Table 2 Pain before and after treatment evaluated by VAS and WOMAC Pain sub-scale.

\begin{tabular}{|c|c|c|c|c|c|c|}
\hline Variable & VAS before & VAS after & $\mathbf{P}$ & WP before & WP after & $\mathbf{P}$ \\
\hline GLOBAL & $8.14 \pm 1.09$ & $2.96 \pm 1.53$ & 0.0000 & $14.88 \pm 7.02$ & $5.55 \pm 6.87$ & 0.0000 \\
\hline $\mathrm{OA} \mathrm{KL} 2^{\circ}$ & $7.0 \pm 2.16$ & $1,5 \pm 2.38$ & 0.0191 & $13.25 \pm 3.59$ & $3.75 \pm 4.34$ & 0.0192 \\
\hline $\mathrm{OA} \mathrm{KL} 3^{\circ}$ & $8.18 \pm 0,65$ & $3 \pm 1.09$ & 0.0000 & $14.75 \pm 2.17$ & $5.5 \pm 1.59$ & 0.0000 \\
\hline $\mathrm{OA} \mathrm{KL} 4^{\circ}$ & $8.71 \pm 0.75$ & $3.71 \pm 1.49$ & 0.0000 & $16.14 \pm 2.91$ & $6.71 \pm 3.19$ & 0.0001 \\
\hline
\end{tabular}

OA, Osteoarthritis; K-L, Kellgren-Lawrence; SD, Standard deviation;VAS,Visual analogical scale;WOMAC,Western ontario and Mc master universities index for osteoarthritis;WP,WOMAC pain

Table 3 Stiffness and function before and after treatment evaluated by WOMAC Stiffness and WOMAC Function sub-scales.

\begin{tabular}{lllllll}
\hline Variable & WS before & WS after & P & WF before & WF after & P \\
\hline GLOBAL & $2.44 \pm 3.33$ & $0.4 \pm 0.4$ & $\mathbf{0 . 0 0 0 0}$ & $44.48 \pm 12.27$ & $15.25 \pm 12.38$ & $\mathbf{0 . 0 0 0 0}$ \\
OA KL $2^{\circ}$ & $1.25 \pm 1.25$ & $0 \pm 0$ & $\mathbf{0 . 1 4 1 1}$ & $28.75 \pm 10.37$ & $6 \pm 9.52$ & $\mathbf{0 . 0 0 6 5}$ \\
OA KL $^{\circ}$ & $3.06 \pm 1.76$ & $0.56 \pm 0.62$ & $\mathbf{0 . 0 0 0 0}$ & $45.12 \pm 10.27$ & $14.12 \pm 9.38$ & $\mathbf{0 . 0 0 0 0}$ \\
OA KL $^{\circ}$ & $1.71 \pm 1.79$ & $0.28 \pm 0.75$ & $\mathbf{0 . 0 4 6 5}$ & $52 \pm 10.14$ & $23.14 \pm 16.32$ & $\mathbf{0 . 0 0 0 5}$ \\
\hline
\end{tabular}

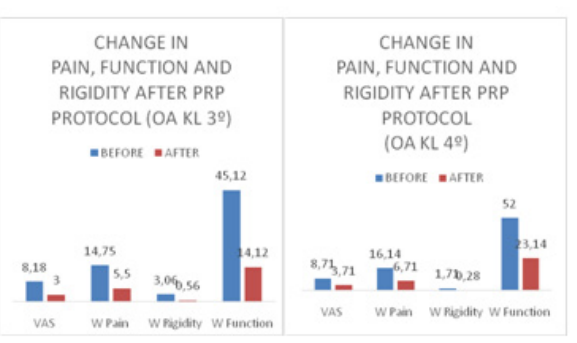

Figure 5 Change in pain, function and rigidity after PRP protocol on $3^{\circ}$ and $4^{\circ}$ grade knee Osteoarthritis.

PRP, Platelet-rich plasma; OA, Osteoarthritis; K-L, Kellgren-Lawrence; VAS, Visual analogical scale; W, WOMAC, Western ontario and Mc master universities index for osteoarthritis;WP,WOMAC pain;WR,WOMAC rigidity;

\section{WFWOMAC function}

\section{Discussion}

To the best of our knowledge, this is the first study that states effectiveness of a standard PRP-protocol on mild, moderate and severe knee OA, and with statistical difference $(\mathrm{p}<0.05)$. PRP demonstrated to be effective in improving pain relief, function and quality of life in all knee OA KL grades ( $2^{\circ}$ to $4^{\circ}$ degree).

The use of stem cells and PRP are becoming a great promise for the healing/regeneration of ligament, tendon, cartilage, and bone. Several case reports and comparative studies have reported improvements on sign and symptoms by pain scores, radiographic and histological evaluation without systemic side effects. ${ }^{21}$ There is new interest in this new treatment option, reflected by many ongoing trials in the last decade. Most of trials are open-label, with focus on knee OA. ${ }^{16}$
Knee OA is a degenerative disease, very prevalent on population and with great impact on morbidity. Conservative treatments tend to relieve pain, recover function and slow or revert the degenerative process. ${ }^{21,32}$ Many studies and case reports state positive findings on pain improvement, ${ }^{21}$ but the halt or reverse of the disease is still to be demonstrated. Several trials have suggested the efficacy of PRP to improve functional outcomes for mild knee OA..$^{23,33}$

The mean age of our sample series was $58.03 \pm 10.91$ years and it is very similar to the most cited studies, like Filardo's (57.5 years), Sánchez (59.7 years), Raeissadat's (59 years), Rayegani's (56.04 years). On the contrary age differs from the series reported by Cerzal (66.4 years) which are older; and those cited by Patel (52.7 years) and Gomeli (53.6 years) which are younger. ${ }^{34}$ Age is an important risk factor for knee OA, although this disease is considered to be multifactorial. $^{3}$

In our study the most frequent grade of knee OA according to K-L scale was $3^{\circ}$ grade $(59.2 \%)$ followed by $4^{\circ}(25.9 \%)$ and $2^{\circ}(14.9 \%)$. This finding is in accordance with other 4 studies from the metaanalysis of Meheaux, where $2^{\circ}(40.7 \%)$ and $3^{\circ}$ grades $(37.9 \%)$ were the most frequent $\mathrm{OA}$ grades, followed by $4^{\circ}(12.6 \%)$ and $1^{\circ}(8.7 \%) .^{18}$ In the same study, Filardo et al. ${ }^{42}$ reported only average severity of knee OA; where $2^{\circ}$ grade was again the most frequent. ${ }^{18}$ Similarly, the meta-analysis from Kanchanatawan mainly considers only patients on $1^{\mathrm{o}}$ and $2^{\mathrm{o}} \mathrm{K}-\mathrm{L}$ grades. ${ }^{34}$ Most of the studies from Dai's meta-analysis considered for treatment $1^{\circ}-3^{\circ} \mathrm{K}-\mathrm{L}$ grades, and only some of them (Gomeli'sl and Sanchez') treated patients with severe OA (K-L $4^{\circ}$ grade). This analysis is very important because most of Randomized Control Trial ${ }^{23}$ have demonstrated effectiveness of PRP in decreasing pain and recovering function ${ }^{35-37}$ especially for younger patients and milder knee $\mathrm{OA},{ }^{38-40}$ but there is only one study that showed benefit of 
PRP even for $3^{\circ}$ grade in knee OA. ${ }^{41}$ Our study contributes to show the efficacy of PRP even in patients with K-L $4^{\circ}$ grade.

In our study, a PRP-protocol of 3 injections has shown effectiveness in improving pain relief, function and rigidity in knee OA patients globally and in all grades of knee OA severity. This is in accordance with most present studies that show moderate quality of evidence supporting the use of PRP on knee OA treatment. ${ }^{6,14,16,18,21-23}$ PRP is capable to improve pain and function measured by VAS and WOMAC. ${ }^{6,23}$ But, when it comes to see whether PRP is effective in all grades of severity, is still a matter of controversy. Wu et al states that most of trials suggest the efficacy of PRP to improve functional outcomes only for mild knee OA. ${ }^{23}$ Spaková concluded that autologous PRP is effective and safe in early knee OA $\left(\mathrm{K}-\mathrm{L} 1^{\circ}, 2^{\circ}\right.$ and $3^{\circ}$ grades). ${ }^{36}$ Moreover, Zlotnicki et al ${ }^{21}$ state that multiple studies suggest that advanced knee OA implies ineffective response to PRP injection therapy. ${ }^{39,42,43}$

Forogh et al. ${ }^{19}$ sustain that some studies have reported an inverse relationship between age and response to treatment; in younger patients the outcome was better than those over 50 years. ${ }^{44}$ FernándezCuadros et al have stated that age is related to knee OA severity and outcomes are dependent on such variable. ${ }^{3,44}$ There is only one study that shows benefit on older patients with even $3^{\circ}$ grade knee OA. ${ }^{41}$ Our present study shows a clear correlation between age and knee severity and for the first time shows statistical significant improvement of PRP-protocol treatment in all K-L grades, even $4^{\circ}$ grade, to the best of our knowledge.

There is controversy on PRP processing; therefore, the variability for its clinical responses. However, despite the technique and formulation discrepancies, intra articular PRP injections are effective in degenerative knees. ${ }^{6}$ With respect to the number of injections and their frequency, most protocols are different. ${ }^{6,18-20,34}$ Almost all of them require three injections without any specific reasons or specific justification. It is believed that the traditional practice of 3 Hyaluronic Acid injections is followed to PRP protocols. ${ }^{19} \mathrm{In}$ fact, no difference in outcomes for pain and function came from having a single or a double injection of PRP, but both provided superior outcomes compared with saline control. ${ }^{23,45}$

Görmeli et al. ${ }^{40}$ compared 3 injections of PRP to one injection of PRP (plus 2 saline injections) or 3 Hyaluronic Acid (HA) injections or 3 saline injections. Three PRP injections showed better scores in EQ-VAS and IKDC at 6 months compared to one single PRP injection or HA injection. ${ }^{22,40}$ Gobbi et al. ${ }^{46}$ showed significant knee OA improvements up to 1 year after PRP injections. ${ }^{22,46}$ Forogh has stated that one-PRP injection diminished pain for a longer term and improved activities of daily life for a short-time duration, better than corticosteroids. ${ }^{19}$

With regard to the quantity of PRP infiltrated, scientist administered volumes that vary from 2 to $8 \mathrm{ml}$, being the most frequent volume $3 \mathrm{ml}$. ${ }^{6,20,34}$ The same controversy exits with respect to spinning; some authors preferred single spinning, ${ }^{35,38,41,45}$ while others prefer double spinning. ${ }^{37,42,6}$

There is controversy about the necessity to an optimal activation method in clinical practice. ${ }^{22}$ By activation, platelets release alpha granules. ${ }^{47}$ The objective to activate PRP is to ensure that growth factors are immediately available. ${ }^{47}$

PRP infiltration technique is safe and only small frequencies of adverse effects are reported (mainly infections and allergic reactions). Pain after injection is greater in PRP when compared to HA infiltration. ${ }^{46}$ In our study one out of 28 patients (3.44\%) presented severe pain and inflammation which lead the patient to quit treatment protocol. No other adverse effects were reported on this series. This confirms that PRP treatment is safe.

Despite positive findings and outcome results, most of those results are inconclusive due to low level-evidence studies, diverse PRP protocols and outcome measures. ${ }^{21}$ The present quasi-experimental before-and-after study, tries to solve such weaknesses, by the use of an stablished protocol, the use of validated outcome measures (VAS and WOMAC Index) and a before-and-after follow-up analysis, all of that gives a good level of evidence, ${ }^{33}$ that let us postulate PRP as a promising option for knee OA treatment. ${ }^{16}$

The importance to show our clinical experience in a series of patients is to provide more clinical evidence of an stablished PRPtreatment protocol on knee OA; because, although there is plenty of current meta-analysis, most of them make reference to only 5 to 6 randomized control trials (RCT). ${ }^{6,16,18,20,22,34}$

As a resume, activated PRP releases cytokines and growth factors, promoting chondrocyte proliferation and differentiation, modulating inflammation and exerting mesenchymal stem cell proliferation. ${ }^{16,18}$ PRP exerts and anti-inflammatory effect by the inhibition of NF$\kappa \beta$ pathway, ${ }^{16,18}$ but also by a decrease in expressing inflammatory enzymes cyclooxygenase 2 and 4 , metalloproteinases and disintegrins. ${ }^{48}$ All those combined PRP effects make this treatment as a potential injectable option in the management of knee OA, improving pain relief and function, as it was demonstrated on this study.

\section{Study limitations}

An important limitation of the study is the small size of the series $(n=27)$. Thirty six months were needed to collect such a number of patients, since this is a prospective study. Another limitation of the study is the absence of control group, because of the limited number of cases. As the effectiveness of PRP on knee OA is supported by several Randomized Control Trials, it was not ethical to deny the intervention, when other conservative non-surgical treatments had already failed. The before-and-after study was designed to solve this specific ethical situation, the absence of control group and to support clinical based-evidence. ${ }^{33,49,50}$ Besides, this type of study tries to evaluate the impact of an intervention, and since there is only one group, no randomization is possible. Despite all previous considerations, this is a kind of experimental study with a good level of evidence; in fact, the Canadian Task Force on Preventive Health Care gives these studies a II-B level of evidence. ${ }^{33,49,50}$ Despite the small size of the series and the absence of control group, those limitations do not affect the results observed in our study.

\section{Conclusion}

PRP Infiltrated in a 3-doses-protocol is effective in the management of knee OA symptoms such as pain and stiffness, improving function and QoL measured by validated outcome scales such as VAS and WOMAC. PRP alleviates knee OA symptoms in all radiological knee 
OA grades, even the most severe ones and with statistical difference.

\section{Acknowledgments}

To Aureliana Gómez Sierra for the collecting of data and preparation of material for intra articular infiltration, to Marĺa del Carmen Martinez de la Riva Molina and to Saturnino Diaz-Trujillo for the article searching.

\section{Conflict of interest}

Authors declare there are no conflicts of interest in publishing the article.

\section{References}

1. Fernández-Cuadros ME. Ozone fundamentals and effectiveness on knee pain: Chondromalacia and knee osteoarthritis. Berlin Lambert Academic Publishing; 2016.

2. Fernández-Cuadros ME, Pérez-Moro OS, Mirón-Canelo JA. Could Ozone Be Used as a Feasible Future Treatment in Osteoarthritis of the Knee? Diversity \& Equality in Health and Care. 2016;13(3):232-239.

3. Fernández-Cuadros ME. Análisis de la Calidad de Vida en pacientes con prótesis de rodilla. Tesis Doctoral. Universidad de Salamanca España; 2013.

4. Dieppe PA, Lohmander LS. Pathogenesis and management of pain in osteoarthritis. Lancet. 2005;365(9463):965-973.

5. Johnson VL, Hunter DJ. The epidemiology of osteoarthritis. Best practice \& research Clinical rheumatology. 2014;28(1):5-15.

6. Dai WL, Zhou AG, Zhang H, et al. Efficacy of Platelet-Rich Plasma in the Treatment of Knee Osteoarthritis: A Meta-analysis of Randomized Controlled Trials. Arthroscopy. 2017;33(3):659-670.

7. Cuadros MEF, Moro OSP, Florin MJA, et al. Ozone improves pain relief function and quality of life in patients with knee osteoarthritis: A prospective quasi-experimental before-after study. Middle East Journal of Rehabilitation and Health. 2017;4(1):e41821.

8. Carr A,J Robertsson O, Graves S, et al. Knee replacement. Lancet. 2012;379(9823):1331-1340.

9. Bijlsma JW, Berenbaum F, Lafeber FP. Osteoarthritis: an update with relevance for clinical practice. Lancet. 2011;377(9783): 2115-2126.

10. Richmond J, Hunter D, Irrgang J, et al. American Academy of Orthopaedic Surgeons clinical practice guideline on the treatment of osteoarthritis (OA) of the knee. J Bone Joint Surg Am. 2010;92(4):990-993.

11. Holt HL, Katz JN, Reichmann WM, et al. Forecasting the burden of advanced knee osteoarthritis over a 10-year period in a cohort of 60-64 year-old US adults. Osteoarthritis cartilage. 2011;19(1):44-50

12. Fernandez-Cuadros ME, Perez-Moro OS, Albaladejo-Florin MJ. Knee Osteoarthritis: Condroprotector Action and Symptomatic Effect of Ozone on Pain Function Quality of Life Minimal Joint Space and Knee Arthroplasty Delay. Middle East Journal of Rehabilitation and Health. 2017;4(1):e43200.

13. McAlindon TE, Bannuru R, Sullivan MC, et al. OARSI guidelines for the non-surgical management of knee osteoarthritis. Osteoarthritis and cartilage. 2014;22(3):363-388.

14. Dhillon MS, Patel S. Role of platelet rich plasma in early osteoarthritis of knee. Journal of Arthroscopy and Joint Surgery. 2015;2(2):55-56.

15.Zhang W, Nuki G, Moskowitz RW, et al. OARSI recommendations for the management of hip and knee osteoarthritis: part III: Changes in evidence following systematic cumulative update of research published through
January 2009. Osteoarthritis Cartilage. 22010;18(4);476-499.

16. Ornetti P, Nourissat G, Berenbaum F, et al. Does platelet-rich plasma have a role in the treatment of osteoarthritis?. Joint Bone Spine. 2016;83(1):3136.

17. Brown GA. AAOS Clinical Practice Guideline: Treatment of Osteoarthritis of the Knee: Evidence $\square$ Based Guideline. J Am Acad Orthop Surg. 2013;21(9):577-579.

18. Meheux CJ, McCulloch PC, Lintner DM, et al. Efficacy of intra-articular platelet-rich plasma injections in knee osteoarthritis: a systematic review. Arthroscopy. 2016;32(3):495-505

19. Forogh B, Mianehsaz E, Shoaee S, et al. Effect of single injection of platelet-rich plasma in comparison with corticosteroid on knee osteoarthritis: a double-blind randomized clinical trial. J Sports Med Phys Fitness. 2015;56(7-8):901-908.

20. Chahla J, Piuzzi NS, Mitchell JJ, et al. Intra-Articular Cellular Therapy for Osteoarthritis and Focal Cartilage Defects of the Knee. J Bone Joint Surg Am. 2016;98(18):1511-1521.

21.Zlotnicki JP, Watson J, Rothrauff BB, et al. Current State for Clinical Use of Stem Cells and Platelet-Rich Plasma. Operative Techniques in Orthopaedics. 2016;26(2):89-97.

22. Lansdown DA, Fortier LA. Platelet Rich Plasma: Formulations Preparations Constituents and Their Effects. Operative Techniques in Sports Medicine. 2016;25(1):7-12.

23. Wu PI, Diaz R, Borg-Stein J. Platelet-rich plasma. Phys Med Rehabil Clin N Am. 2016;27:825-853.

24. Xie X, Zhang C, Tuan RS. Biology of platelet-rich plasma and its clinical application in cartilage repair. Arthritis Res Ther. 2014;16(1):204.

25. Sheth U, Simunovic N, Klein G, et al. Efficacy of autologous platelet-rich plasma use for orthopaedic indications: a meta-analysis. J Bone Joint Surg Am. 2012;94(4):298-307.

26. Cho HJ, Chang CB, Yoo JH, et al. Gender differences in the correlation between symptom and radiographic severity in patients with knee osteoarthritis. Clin Orthop Relat Res. 2010;468(7):1749-1758.

27. Bellamy N, Buchanan WW, Goldsmith $\mathrm{CH}$, et al. Validation study of WOMAC: a health status instrument for measuring clinically important patient relevant outcomes to antirheumatic drug therapy in patients with osteoarthritis of the hip or knee. J Rheumatol. 1988;15(12):1833-1840.

28. Wright RW. Knee sports injury outcome measures. $J$ Knee Surg. 2005;18(01):69-72.

29. Kellgren JH, Lawrence JS. Radiological assessment of osteoarthrosis. Annals of the rheumatic diseases. 1957;16(4):494

30. Boegård T, Jonsson K. Radiography in osteoarthritis of the knee. Skeletal radiology. 1999;28(11):605-615.

31. Angst F, Aeschlimann A, Stucki G. Smallest detectable and minimal clinically important differences of rehabilitation intervention with their implications for required sample sizes using WOMAC and SF $\square 36$ quality of life measurement instruments in patients with osteoarthritis of the lower extremities. Arthritis Rheum. 2001;45(4):384-391.

32. Kapoor M, Martel-Pelletier J, Lajeunesse D, et al. Role of proinflammatory cytokines in the pathophysiology of osteoarthritis. Nat Rev Rheumatol. 2013;7(1):33-42.

33. Mirón-Canelo JA. Sistema de Información Sanitaria. Indicadores de Salud Bienestar y Calidad de Vida. En: Guías para la elaboración de trabajos científicos Grado Máster y Posgrado. Salamanca España. 2013

34. Kanchanatawan W, Arirachakaran A, Chaijenkij K, et al. Short-term

Citation: Fernández-Cuadros ME, Pérez-Moro OS,Albaladejo-Florín MJ, et al. Effectiveness of platelet-rich plasma (PRP) on pain, function and quality of life in knee osteoarthritis patients: a before-and-after study and review of the literature. MOJ Orthop Rheumatol. 20I8;10(3):202-208. 
outcomes of platelet-rich plasma injection for treatment of osteoarthritis of the knee. Knee Surg Sports Traumatol Arthrosc. 2016;24(5):1665-1677.

35. Vaquerizo V, Plasencia MÁ, Arribas I, et al. Comparison of intraarticular injections of plasma rich in growth factors (PRGF-Endoret) versus Durolane hyaluronic acid in the treatment of patients with symptomatic osteoarthritis: a randomized controlled trial. Arthroscopy. 2013;29(10):1635-1643.

36. Spaková T, Rosocha J, Lacko M, et al. Treatment of knee joint osteoarthritis with autologous platelet-rich plasma in comparison with hyaluronic acid Am J Phys Med Rehabil. 2012;91(5):411-417.

37. Raeissadat SA, Rayegani SM, Hassanabadi H, et al. Knee osteoarthritis injection choices: platelet-rich plasma (PRP) versus hyaluronic acid (a oneyear randomized clinical trial). Clin Med Insights Arthritis Musculoskelet Disord. 2015;8:1-8.

38. Sánchez M, Fiz N, Azofra J, et al. A randomized clinical trial evaluating plasma rich in growth factors (PRGF-Endoret) versus hyaluronic acid in the short-term treatment of symptomatic knee osteoarthritis. Arthroscopy. 2012;28(8):1070-1078.

39. Kon E, Mandelbaum B, Buda R, et al. Platelet-rich plasma intra-articular injection versus hyaluronic acid viscosupplementation as treatments for cartilage pathology: from early degeneration to osteoarthritis. Arthroscopy. 2011;27(11):1490-1501.

40. Görmeli G, Görmeli CA, Ataoglu B, et al. Multiple PRP injections are more effective than single injections and hyaluronic acid in knees with early osteoarthritis: a randomized double-blind placebo-controlled trial. Knee Surg Sports Traumatol Arthrosc. 2017; 25(3):958-965.

41. Cerza F, Carnì S, Carcangiu A, et al. Comparison between hyaluronic acid and platelet-rich plasma intra-articular infiltration in the treatment of gonarthrosis. Am J Sports Med. 2012;40(12):2822-2827.
42. Filardo G, Kon E, Di Martino A, et al. Platelet-rich plasma vs hyaluronic acid to treat knee degenerative pathology: study design and preliminary results of a randomized controlled trial. BMC Musculoskelet Disord. 2012;13(1):229.

43. Khoshbin A, Leroux T, Wasserstein D, et al. The efficacy of platelet-rich plasma in the treatment of symptomatic knee osteoarthritis: a systematic review with quantitative synthesis. Arthroscopy. 2013;29(12): 2037-2048.

44. Fernandez-Cuadros ME, Perez-Moro OS, Miron-Canelo JA. Age and Comorbidities Affect Quality of Life in Patients With Osteoarthrtitis and Knee Replacement. Middle East Journal of Rehabilitation and Health. 2016;3(4).

45. Patel S, Dhillon MS, Aggarwal S, et al. Treatment with platelet-rich plasma is more effective than placebo for knee osteoarthritis a prospective double-blind randomized trial. Am J Sports Med. 2013;41(2):356-364.

46. Gobbi A, Karnatzikos G, Mahajan V, et al. Platelet-rich plasma treatment in symptomatic patients with knee osteoarthritis: preliminary results in a group of active patients. Sports Health. 2012;4(2):162-172.

47. Li Z, Delaney MK, O’brien KA, et al. Signaling during platelet adhesion and activation. Arterioscler Thromb Vasc Biol. 2010;30(12):2341-2349.

48. Kabiri A, Esfandiari E, Esmaeili A, et al. Platelet-rich plasma application in chondrogenesis. Adv biomed res. 2014;3:138.

49. Manterola C, Otzen T. Estudios Experimentales 2 Parte: Estudios CuasiExperimentales. International Journal of Morphology. 2015;33(1):382387

50. Ray S, Fitzpatrick S, Golubic R, et al. Oxford Handbook of Clinical and Healthcare Research. UK: Oxford; 2016. 